\title{
Investigation on AC breakdown of vegetable oils with insulated electrodes
}

\begin{abstract}
Most of power transformer design today is still using oil filled type which laminated iron core with paper insulated copper conductors. Oil is an important part in power transformers as electrical insulation, information carrier and cooling medium. Besides oil, paper insulation also plays important role as dielectric material of transformer. The influence of oil especially vegetable-based oil and paper insulation on the dielectric strength of transformer oil has attracted many researchers to do investigation. This paper presents a study of the quasiuniform field AC breakdown voltages under bare electrodes, paper insulation for barrier and covered electrodes (to replicate the transformer windings) of Palm Oil (PO), Coconut Oil (CO) and Mineral Oil (MO). The data obtained from all tests were analyzed using Gaussian and Weibull distribution to determine the withstand voltages for each type of oils. The experiments were using test cell as specified by IEC 156 with $2.5 \mathrm{~mm}$ gap distance under uniform field.
\end{abstract}

Keyword: Power transformer; Vegetable oil; AC breakdown voltage; Uniform filed; Kraft paper 